\title{
INTEGRATION OF DESIGN AND ASSEMBLY USING AUGMENTED REALITY
}

\author{
Juha Sääski ${ }^{1}$, Tapio Salonen ${ }^{1}$, Mika Hakkarainen ${ }^{1}$, Sanni Siltanen ${ }^{1}$, Charles \\ Woodward $^{1}$, Juhani Lempiäinen ${ }^{2}$

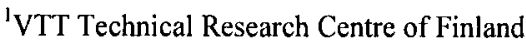 \\ ${ }^{2}$ Deltatron Ltd \\ Juhani Lempiäinen \\ Deltatron Ltd \\ Soidintie 14, Helsinki \\ FI-00700 Helsinki, Finland \\ Tel: $+358-9-3452660$ \\ Fax: $+358-9-3454297$ \\ jle@deltatron.fi \\ www.deltatron.fi \\ Juhani Lempiäinen \\ Deltatron Ltd \\ Soidintie 14, Helsinki \\ FI-00700 Helsinki, Finland \\ Tel: $+358-9-3452660$ \\ Fax: +358-9-3454297 \\ jle@deltatron.fi \\ www.deltatron.fi
}

\begin{abstract}
This paper presents a methodology and a system for augmented reality aided assembly work. We concentrate in particular on the requirements on information processing and data flow for implementing augmented assembly systems in real life production environments. A pilot case with an augmented assembly task at the Finnish tractor company Valtra is described.
\end{abstract}

Keywords augmented reality, assembly work, assembly instruction, PDM, CAD, design for assembly

\section{Introduction}

This paper presents augmented reality (AR) system architecture for assisting assembly work by visual information superimposed on the physical assembly parts. Such AR methods are particularly well suited for complex, short manufacturing se-

Please use the following format when citing this chapter:

Sääski, J., Salonen, T., Hakkarainen, M., Siltanen, S., Woodward, C., Lempiäinen, J., 2008, in IFIP International Federation for Information Processing, Volume 260, Micro-Assembly Technologies and Applications, eds. Ratchev, S., Koelemeijer, S., (Boston: Springer), pp. 395-404. 
ries or in a customized production factory environment. Each individual product may have a slightly different configuration: the order of assembling parts may vary for different products and/or the number of phases in the assembly line may be large. The traditional approach is to use assembly drawings (blueprints) and possibly instruction manuals with guiding pictures to describe the content of each work task. As the assemblies become even smaller, the need for guiding the worker with all available tools becomes increasingly important. The AR system can also reduce assembly times, accelerate learning of the assembly tasks and provide more quality assurance to the factory floor.

The emphasis in this paper is on content authoring for AR. Our ultimate goal is to automate the augmented assembly content creation pipeline, starting from sales/ordering systems up to product specific assembly instructions displayed on mobile hardware to the workers at the production line. The specific focus in this paper is on integrating design for assembly (DFA) software tools to the design systems (CAD/PDM/PLM). The main objectives are to develop a content creation process from design systems via DFA to AR based assembly instructions, and to show the natural links between DFA work and AR content authoring.

The organization of this paper is as follows. First we present a brief overview of related work on AR assisted assembly research. A brief overview of augmented reality technology is provided next. The main body of the article then discusses 3D CAD and assembly information data flow to content creation and authoring for AR. The two last sections present our first experiments and results, as well as conclusions of the work so far.

\section{Related work}

Augmented reality based technology is closely linked to different kinds of display systems, especially so-called head-mounted displays (HMD). The HMD display technology has been available for many years (Furness 1969), the traditional application being in military field. Examples of some more recent display devices are shown in Fig. 1.
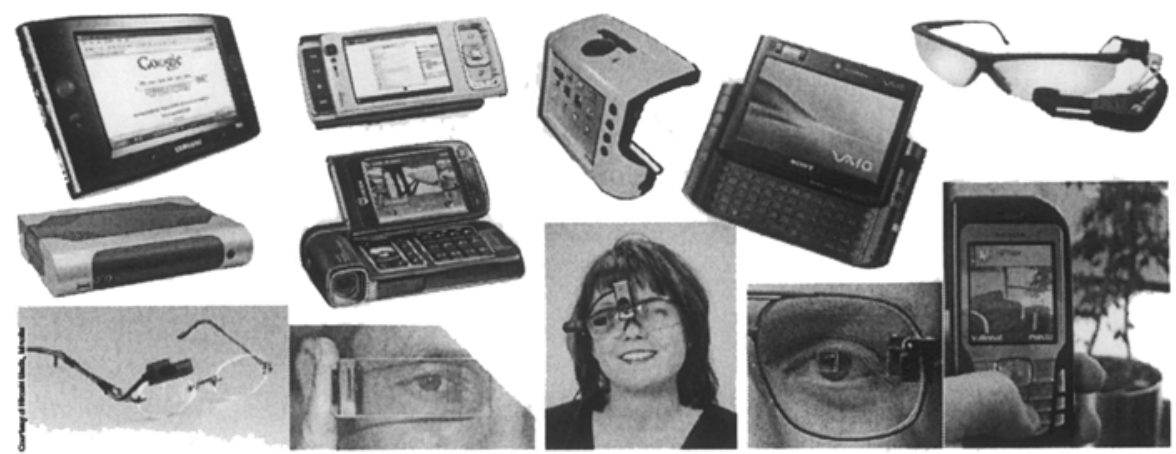

Fig. 1. Examples of augmented reality display devices: hand held units and see through HMD. 
One of the first projects dealing with manufacturing was launched by Boeing in which Claudell and Mizell (Claudell and Mizell 1992) described the challenges in aircraft manufacturing. "Much of the information is derived from engineering designs for parts and processes stored in CAD systems. In many cases, this information comes to the factory floor in the form of assembly guides, templates, drawings, wiring lists, and location markings on sheet metal". "A significant source of expense and delay in aircraft manufacturing comes from the requirement to mirror changes in the engineering design in the guides, templates and so on used to control the manufacturing process. If manufacturing workers were able to directly access digital CAD data when performing manufacturing or assembly operations, several sources of expense and error would be eliminated". Their concept was to provide a "see-thru" display to the factory worker, and use this device to augment the worker's visual field of view with dynamically changing information. In their demonstration system the challenges were to align real and virtual objects with each other and also the capacity of portable computing unit allowing only representing simple graphics in real time. Today we are witnessing huge improvement in mobile computing units and graphics; also the recent HMD development is now led by gaming industry with the so called i-glasses, providing good full screen picture resolution with reasonable pricing.

Recently ARVIKA (www.arvika.de) was a large industrial AR project in Germany 1999-2003, and it consisted of an industrial consortium led by Siemens. The goal was to develop prototypes of portable and fixed location AR systems for development, production and service in the automotive and aircraft industry, both in systems and mechanical engineering. Participants included: automobile manufacturers such as Audi, BMW, DaimlerChrysler, Ford and Volkswagen; aircraft manufacturers such as EADS and Airbus; equipment manufacturers such as MicroVision, Physoptics and Zeiss. ARTESAS (www.artesas.de) (2004-2006) aimed at the exploration and evaluation of augmented reality base technologies for applications in industrial service environments. The project was based on the results of the ARVIKA project. On-going AR research projects for manufacturing industries in Europe are e.g. SmartFactory (www.smartfactory-kl.de), Wearit@Work (www.wearitatwork.com) and Ultra (www.ist-ultra.org). Augmented reality is also explored in many universities and research institutes in U.S.A and Asia.

\section{Augmented reality technology}

Augmented reality systems combine digital information and real world in a way that a user experiences this as a whole. An important property is especially that virtual objects are located to the right place and position. AR system follows dynamically the user's point of view and keeps virtual objects aligned with real world objects. The basic components in AR applications are a display, a camera and a computer with application software (Azuma et al 2001). Various different kinds of 
hardware can be used to implement this, e.g. camera phones, PDAs, laptops, HMDs etc.

Typically, a camera is attached to the display device which shows the real time camera view "through" its screen. To determine the relation between real and virtual worlds, computer vision techniques are used to find (track) a marker in the camera image and determine the position and the pose of the camera relative to it. Once the position and the pose of the real camera are known, a digital 3D-model can be exactly overlain on (or near) the marker in the camera image. Thus the user experiences video see-through augmented reality, seeing the real world through the real time video with virtual models. Figure 2 summarizes the tracking and display process.

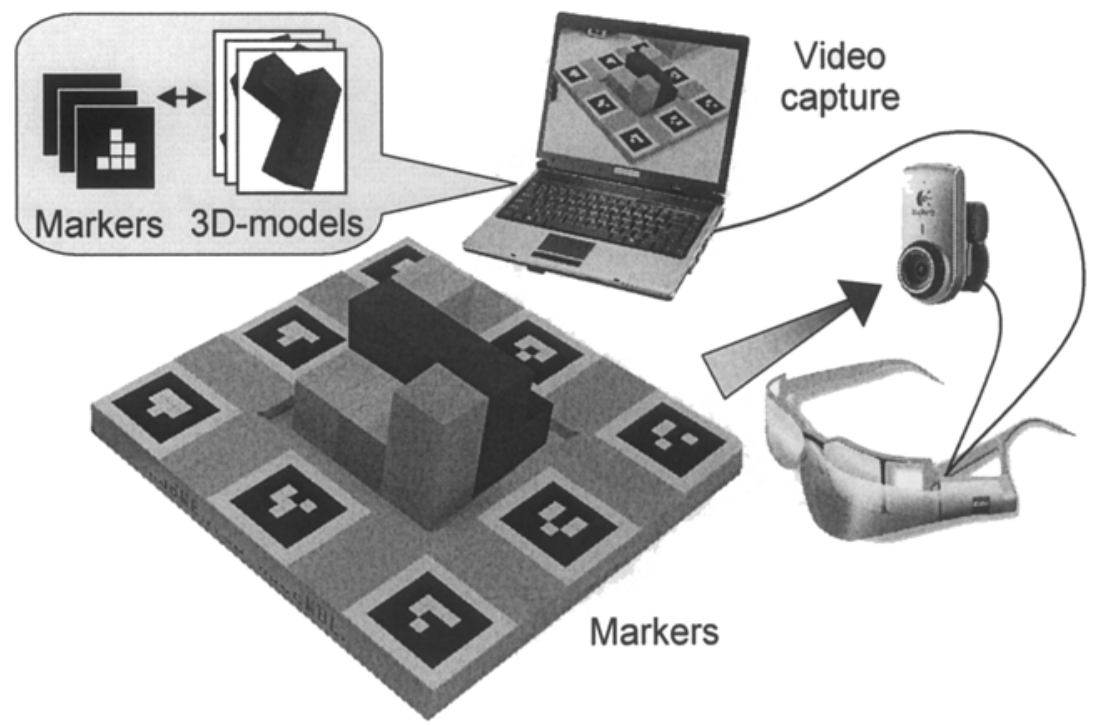

Fig. 2. The augmented reality tracking and display process: the computer-generated graphical augmentation is integrated in the user's view of the real world.

AR has been studied for years, the focus being in hardware technology and not the usability. Thereafter the AR systems have been clumsy, that is heavy and big. However, the rapid development of mobile devices (handhelds) has lead to small units with enough processing capacity and long lasting batteries to enable lightweight mobile AR systems. Recently PDAs (Pasman and Woodward 2003), camera phones (Henrysson et al 2005, Rohs 2006) and miniature PCs (Honkamaa et al 2007) have been successfully used in AR applications. Mobile augmented reality is considered one of the most promising emerging technologies (Jonietz 2007) "most likely to alter industries, fields of research, and even the way we live". 


\section{Augmented Assembly}

One of the biggest challenges for utilisation of AR technology in manufacturing industry is re-use of existing product data. Product data is stored in PDM/PLM systems and these systems include all relevant product data (3D geometry, product structure, simulation results, part fabrication plan, assembly plan etc.). The information should be retrieved from PDM/PLM systems into forms suitable for AR display as automatically as possible; cf. (Matysczock and Ebbesmeyer 2004). Besides finding suitable 3D data representations and conversion methods for AR use, also the assembly related guidance information (annotations, animations etc.) should be considered. Our approach is to exploit ISO 10303 (ISO 10303) definitions for product data representation. However, there is a lack of semantics dealing with representing with AR type of information. Heilala (Heilala et al 2007) have reviewed the latest possibilities integrating information in manufacturing industry.

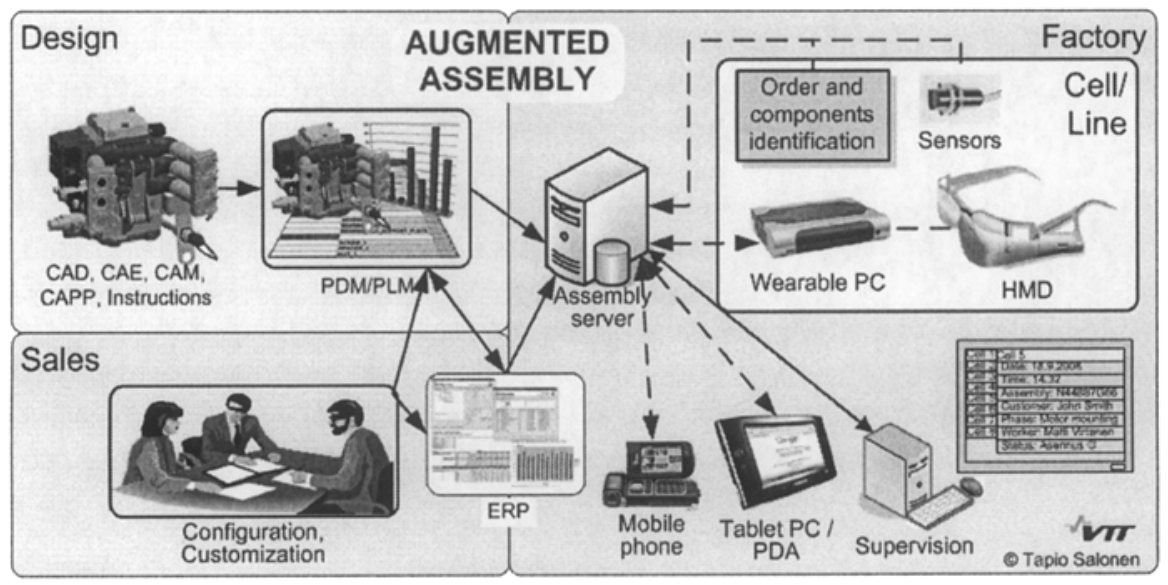

Fig. 3. The proposed augmented reality based information processing architecture.

AR based manufacturing instructions affect different information processing systems of the company in many ways. In order to implement augmented reality based assembly instructions we propose the information processing architecture shown in Fig. 3. The majority of the product data is created in design systems and stored to PDM/PLM system. Sales configure and customise the individual product, for instance. That is also stored to the PDM/PLM system, from which AR based instructions will be created as described below. The ERP system controls production planning, and assembly server, which manages augmented instructions to the worker.

Figure 4 shows the proposed methodology how the AR instructions are created from the product's 3D model. First the CAD model is exported to standard STEP (ISO 10303 1994) format file, that includes the product structure and 3D models of the parts. Because of the designer's preferences, company specific part libraries and features of typical CAD systems the generated product structure usually does not 
conform to the real parts to be assembled in assembly line. The design structure of the product is not equal to the manufacturing assembly structure of the product. Therefore, the assembly structure (i.e. the definition of the assembled parts) and work phases have to be re-configured.

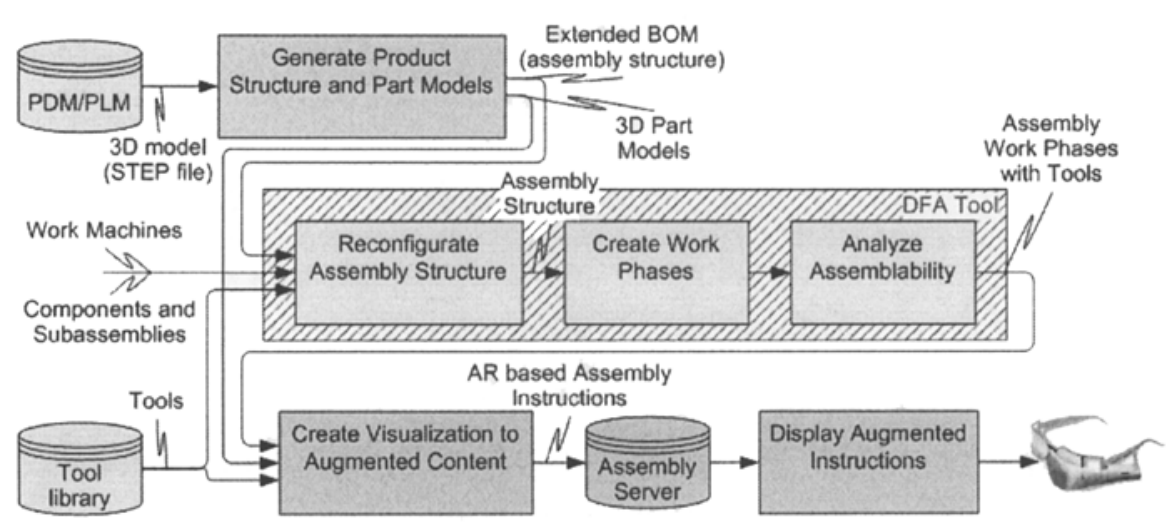

Fig. 4. Creation of AR based assembly instruction using the 3D product model.

As described in (Salmi and Lempiäinen 2005) assemblability analysis can be a very effective tool in authoring the assembly description for the worker. This includes 1) the right and natural component order in a subassembly, 2) use of specific assembly tools for the components, and 3) the special actions needed to be carried out at this point. All these three characteristics are the key elements in the traditional assemblability analysis and suddenly they are equally the main interest for AR authoring in assembly work.

The right assembly order and instructions and hints are generated normally in the late prototype/pre-production phase of the product development. Now with these described AR tools this production documentation can be generated in an early phase and with automated means. This reduces the need for extra written assembly instruction sheets and documented pictures/photos in which assembly sequence these instructions will be applied. The other benefit is the semi-automated assemblability analysis of the product. This analysis will point out any special problematic assembly sequence where some revised constructions can be applied.

In this study the DFA-Tool ${ }^{\mathbb{B}}$ assemblability analysis software is used to carry out the assembly analysis task. The extended Bill Of Materials (BOM) that includes design structure, cf. Fig 4, will be imported to DFA-Tool. The analysis will be carried out there with the reconfiguration the assembly structure, the generation of specific actions, tools and right component assembly order. Fig 5 shows a view of DFATool. Finally the assembly work phases and the 3D part models are exported for augmentation, cf. "Create Visualization to Augmentation Content" in Fig 4. The visual instructions and possibly some text notes are generated using 3D models of assembly tools and components. 


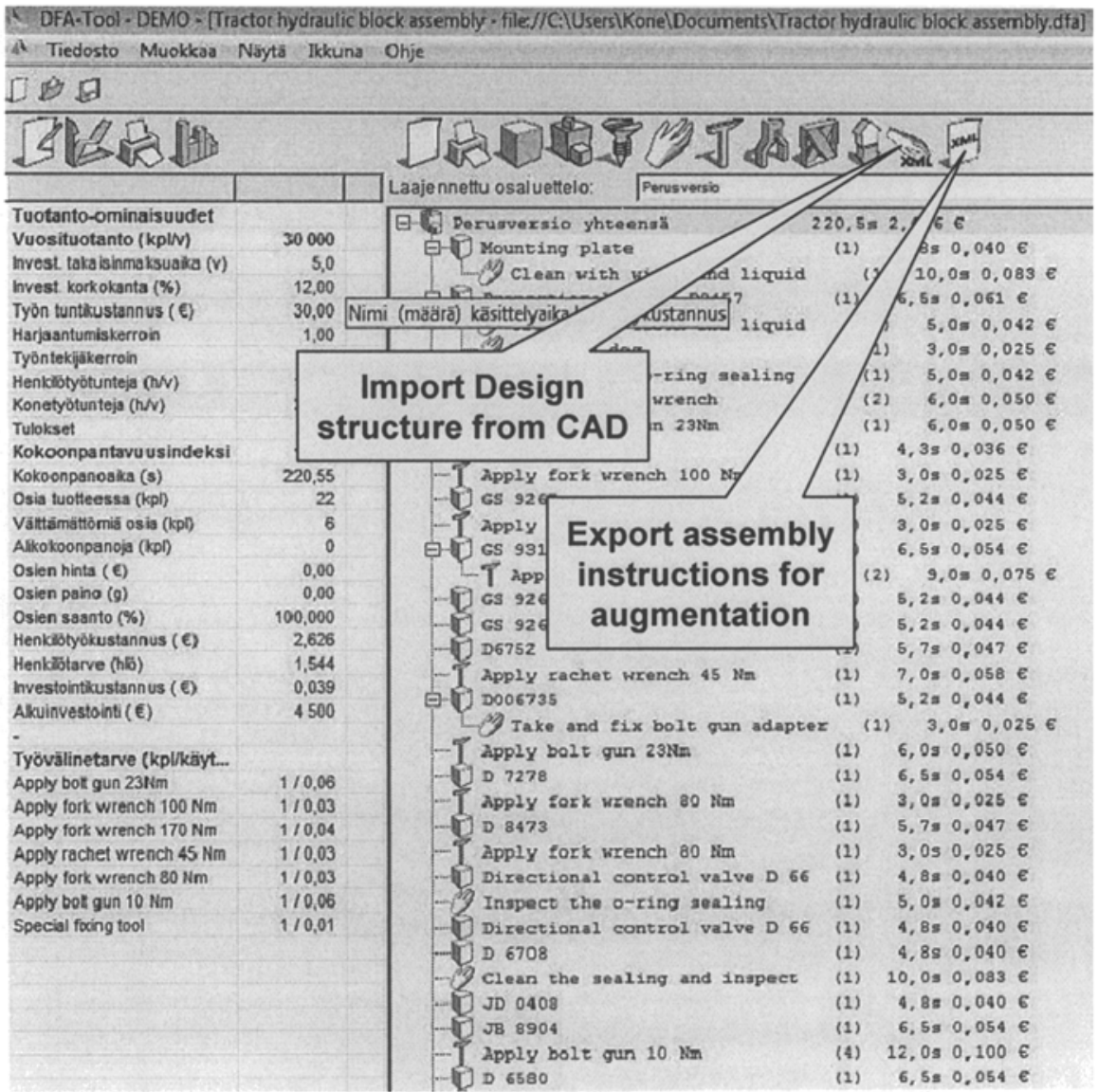

Fig. 5. Augmentation content of a hydraulic block created by DFA analysis tool.

\section{Experiments and results}

In the preliminary tests we have used a simplified assembly task that simulates real assembly work. The task is to put parts in a 3D puzzle box as shown in Fig. 2. At each stage the system shows to the user which part to pick and animates how to place it in the box. The user follows the instructions and puts the parts piece-bypiece according to augmented instructions at the right place and in the right order. The task is real-enough using the actual devices (HMD, camera, etc) and it also serves for our preliminary testing of the content creation components and data flow. User experiences with this test bed system have been most encouraging (Salonen et 
al. 2007, Sääski et al. 2007); cf. also similar work by (Pathomaree and Charoenseang 2005).

Our industrial pilot case focuses on the assembly of a tractor accessory's power unit at the Finnish tractor factory Valtra Plc. The CAD model of the finished block, our case study, is shown left in Fig. 6. Markers on the rotating metal plate are used for tracking the physical unit's orientation. Assembled parts are shown to the worker task by task according to the work phase instructions, overlain on the physical unit. We are investigating alternatives for display devices, from static and hand held PCs to HMDs, as well as multiple camera systems for enhanced accuracy; cf. (Sääski et al. 2007). The preliminary system already includes most of the above described components for automatic data flow from CAD systems to DFA and augmentation; however integration of sales and ERP remains for future work.
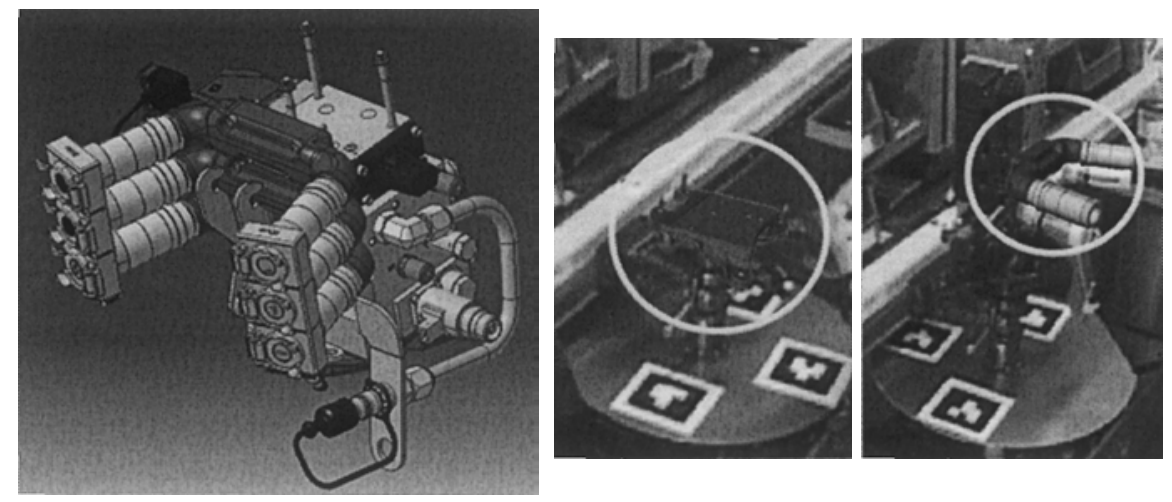

Fig. 6. 3D CAD model of finished hydraulic block assembly (Courtesy of Valtra Plc). On the center the first part is assembled to the fixture. On the right the real parts are assembled, and the worker can see the next component as a virtual part.

\section{Conclusions}

AR-technology (hardware and software) and price have matured to the level that manufacturing industry is starting to seek applications in real factory environments. There have been large industrial research projects with good and promising results. AR-technology has been studied mostly how to display assembly information, but only a few studies are dealing with integration of AR related information with current PDM systems used in industry. In this paper we show a method to retrieve assembly knowledge to AR-system. Our approach relies on usage of ISO 10303 (known as STEP), so that smooth information flow can be realized. Our preliminary tests with $3 \mathrm{D}$ puzzle show that $\mathrm{AR}$ is promising technology at least in laboratory environment. In this on-going project the industrial case is used to test our assump- 
tions with the real case in the factory, and with the real assembly tasks and assembly workers.

As assembly constructions in the future will be even smaller in size and have even more functions, it is important that right assembly tools and assembly guidance are immediately available for every assembly operator in synchronization when a new product version is created. We feel these described techniques with the fast development of light weight wearable display devices will make a huge improvement for the quality of both the augmented assembly constructions and their physical counterparts as well.

DFA-Tool ${ }^{\circledR}$ is a registered trade mark of Deltatron Ltd, see www.dfa-tool.eu

\section{Acknowledgements}

This contract based research project is funded by Tekes, The Finnish Agency for Technology and Innovation through its manufacturing technology programme SISU 2010. Key industrial companies also cover partially the costs of this work. The research team thanks for the contributions.

\section{References}

1. Azuma R, Baillot Y, Behringer R, Feiner S, Julier S, and MacIntyre B, (2001). Recent advances in augmented reality, IEEE Computer Graphics and Applications, 21 (2001), no. $6,34-47$, issn $0272-1716$.

2. Caudell TP, Mizell DW, (1992). Augmented reality: an application of heads-up display technology to manual manufacturing processes. Proceedings of the Twenty-Fifth Hawaii International Conference on System Sciences. Volume ii, page(s):659 - 669

3. Furness T, (1969). Helmet-mounted displays and their aerospace applications. National aerospace electronic conference, Dayton, OH, U.S.A.

4. Heilala J, Helaakoski H, Peltomaa I (2007). Smart Assembly - data and model driven. Fourth International, Precision Assembly Seminar, IPAS'2008. Chamonix, France, 1013 February 2008 . Submitted for publication.

5. Henrysson A, Billinghurst M, Ollila M, (2005). Virtual object manipulation using a mobile phone", Proc. 15th International Conference on Artificial Reality and Telexistence (ICAT 2005), Dec 5th 8th, 2005, Christchurch, New Zealand, pp. 164-171.

6. Honkamaa P, Siltanen S, Jäppinen J, Woodward C, Korkalo O, (2007). Interactive outdoor mobile augmentation using markerless tracking and GPS. To appear in Proc. VRIC - Laval Virtual 2007.

7. ISO 10303-203 (1994). Industrial automation systems and integration -- Product data representation and exchange -- Part 203: Application protocol: Configuration controlled 3D designs of mechanical parts and assemblies. Geneva, Switzerland: ISO. $581 \mathrm{p}$

8. Jonietz E, (2007). Augmented Reality: Special Issue 10 Emerging Technologies 2007, MIT Technology Review, March/April 2007. 
9. Matysczock C, Ebbesmeyer P, (2004). Efficient creation of augmented reality content by using an intuitive authoring system, Proc. DETC'04, Salt Lake City, Utah, USA (2004), pp. 53-60.

10. Pahl G, Beitz W, (2003). Engineering Design. A systematic approach. 2nd edition. London: Springer Verlag. $544 \mathrm{p}$.

11. Pang Y, Nee AYC, Ong SK, Yuan ML and Youcef-Toumi K, (2006). Assembly feature design in an augmented reality environment", Assembly Automation Journal, Vol. 26, No. 1, 34-43 (2006).

12. Pasman W, Woodward C, (2003). Implementation of an augmented reality system on a PDA, Proc. The Second IEEE and ACM International Symposium on Mixed and Augmented Reality (ISMAR 2003), Tokyo, Japan, October 2003, pp. 276-277.

13. Pathomaree N, Charoenseang S, (2005). Augmented reality for skill transfer in assembly task, 2005 IEEE International Workshop on Robotics and Human Interactive Communication, pp. 500-504.

14. Rohs M, (2006). Marker-Based Embodied Interaction for Handheld Augmented Reality Games, Proceedings of the 3rd International Workshop on Pervasive Gaming Applications (PerGames) at PERVASIVE 2006, Dublin, Ireland, May 2006

15. Salmi T, Lempiäinen J (2006). First stemps in Integrating Microassembly Features into Industrially Used DFA Software, Proceedings of the IFIP Third International Precision Assembly Seminar IPAS 2006, Bad Hofgastein, Austria, 19-21 February, 2006, pp 149154.

16. Salonen T, Sääski J, Hakkarainen M, Kannetis T, Perakakis M, Siltanen S, Potamianos A, Korkalo O, Woodward C, (2007). Demonstration of Assembly Work Using Augmented Reality, Proceedings of the ACM International Conference on Image and Video Retrieval, 2007, Amsterdam, The Netherlands, 9-11 July 2007, pp. 124-126.

17. Sääski J, Salonen T, Siltanen S, Hakkarainen M, Woodward C, (2007). Augmented Reality Based Technologies For Supporting Assembly Work, Proceedings of $6^{\text {th }}$ Eurosim Congress on Modelling and Simulation. Vol.2, 2007, Ljubljana, Slovenia, 9-13 September 2007. 